\title{
Virulence variation among epidemic and non-epidemic strains of Saint Louis encephalitis virus circulating in Argentina
}

\author{
María Elisa Rivarola/ ${ }^{+}$, Laura Beatriz Tauro, Guillermo Albrieu Llinás, Marta Silvia Contigiani \\ Laboratorio de Arbovirus, Instituto de Virología Dr JM Vanella, Facultad de Ciencias Médicas, \\ Universidad Nacional de Córdoba, Córdoba, Argentina
}

\begin{abstract}
Saint Louis encephalitis virus caused an outbreak of febrile illness and encephalitis cases in Córdoba, Argentina, in 2005. During this outbreak, the strain CbaAr-4005 was isolated from Culex quinquefasciatus mosquitoes. We hypothesised that this epidemic variant would be more virulent in a mouse model than two other non-epidemic strains (78V-6507 and CorAn-9275) isolated under different epidemiological conditions. To test this hypothesis, we performed a biological characterisation in a murine model, including mortality, morbidity and infection percentages and lethal infection indices using the three strains. Mice were separated into age groups (7,10 and 21-day-old mice) and analysed after infection. The strain CbaAr-4005 was the most infective and lethal of the three variants, whereas the other two strains exhibited a decreasing mortality percentage with increasing animal age. The strain CbaAr4005 produced the highest morbidity percentages and no significant differences among age groups were observed. The epidemic strain caused signs of illness in all inoculated animals and showed narrower ranges from the onset of symptoms than the other strains. CbaAr-4005 was the most virulent for Swiss albino mice. Our results highlight the importance of performing biological characterisations of arbovirus strains likely to be responsible for emerging or reemerging human diseases.
\end{abstract}

Key words: arbovirus - flavivirus - Saint Louis encephalitis virus - biological characterisation - virulence

Saint Louis encephalitis virus (SLEV) (genus Flavivirus, family Flaviviridae), a member of the Japanese encephalitis virus (JEV) complex, is widely distributed throughout the Americas, from Canada to Argentina. In South America, SLEV causes an emerging arbovirosis, with febrile illness and encephalitis cases having been reported in Argentina and Brazil (Monath 1980, Rocco et al. 2005, Díaz et al. 2012).

SLEV reemerged in the central region of Argentina in 2002 (Spinsanti et al. 2003); since then, outbreaks were reported in Córdoba (2005) (Spinsanti et al. 2008), Entre Ríos (2006), Buenos Aires (2010) and San Juan (2011) (Seijo et al. 2011). In 2005, 47 laboratory-confirmed and probable clinical cases of SLEV infection, including nine fatalities, were reported in central Córdoba province (Spinsanti et al. 2008).

In the United States of America (USA), the transmission cycles of SLEV are maintained by mosquito vector species of the genus Culex (Culex quinquefasciatus, Culex tarsalis and Culex nigripalpus) and bird host species, such as columbiformes (Mourning doves - Zenaida macroura) and passeriformes (House finches - Carpodacus mexicanus; House sparrows - Passer domesticus). Although SLEV is widely distributed in

doi: 10.1590/0074-0276130475

Financial support: PID MINCyT (Cba 113/11), SECyT (UNC 162/12), CONICET (Pip 2010)

+ Corresponding author: mariaelisarivarola@gmail.com

Received 25 September 2013

Accepted 19 February 2014 the American continent, little is known about its ecology outside the USA (Reisen 2003). In Argentina, it was suggested that $C x$. quinquefasciatus mosquito would act as a main vector, whereas Picui ground dove (Columbina picui) and Eared dove (Zenaida auriculata) would be important hosts in urban and rural environments (Díaz et al. 2008, Díaz 2009). In addition, Sabattini et al. (1998) postulated an alternative transmitting rodent-mosquito cycle for SLEV strains isolated from Mus musculus and Calomys spp mice.

SLEV shows a high genetic diversity throughout the American continent (Bowen et al. 1980, Trent et al. 1980, Mitchell et al. 1983, Rodrigues et al. 2010). Based on phylogenetic studies using the complete open reading frame sequence $(10,236 \mathrm{bp})$ and the envelope coding gene, SLEV has been classified into eight genotypes, each one with specific patterns of geographical distribution (Rodrigues et al. 2010). In Argentina, different strains have been isolated in endemic and epidemic scenarios. The endemic strains CorAn-9275 and CorAn9124 (genotype VII) were isolated from M. musculus and Calomys spp rodents, respectively, in Córdoba province in 1967 (Sabattini et al. 1998). The endemic strain 78V-6507 (genotype V) was isolated from Cx. quinquefasciatus mosquitoes in Santa Fe province in 1978 (Sabattini et al. 1998) and epidemic strains (CbaAr-4005 and CbaAr-4006, both genotype III) were isolated from $C x$. quinquefasciatus mosquitoes collected from around the house of SLEV-confirmed patients (Díaz et al. 2006), during an unprecedented outbreak in 2005. Likewise, SLEV shows an important biological variability, described as differential neuroinvasiveness in monkeys and rodents or viraemia in birds (Bowen et al. 1980, Monath 1980, Trent et al. 1980). No biological characterisation 
assays have still been performed using SLEV CbaAr4005 , which was recently isolated during an outbreak in Córdoba city.

We hypothesised that in a mouse model the SLEV epidemic variant $\mathrm{CbaAr}-4005$ is more virulent than two non-epidemic strains, 78V-6507 and CorAn-9275, isolated under different epidemiological conditions. To test this hypothesis, we performed biological characterisations of the epidemic strain CbaAr-4005 and non-epidemic strains CorAn-9275 and 78V-6507, using Swiss albino mice. Qualitative and quantitative biological markers of virulence were identified and compared between the viral strains. Our study will help us to understand why strain CbaAr-4005 (and not CorAn-9275 neither 78V-6507) caused a disease outbreak in Córdoba, Argentina, during 2005.

\section{MATERIALS AND METHODS}

Viruses - All SLEV isolates used in our study (CbaAr-4005, 78V-6507 and CorAn-9275) are available at the Laboratory of Arboviruses, in the Virology Institute of the National University of Córdoba, Argentina. The SLEV strains used in this study were derived from nine rounds of propagation through suckling mouse brains. Seed stocks for these experiments were prepared by suspending 10\% w/v Swiss albino mice brain in Eagle's minimum essential medium (MEM) supplemented with $10 \%$ foetal bovine serum and $1 \%$ gentamicin. Virus stock titres were determined by standard Vero cell plaque assay and expressed as plaque forming units per millilitres (p.f.u./mL) (Contigiani \& Sabattini 1977).

Mice Rockefeller Swiss albino mice, strain W1 (R-W1), of seven, 10 and 21 days of age were used; all experiments were performed in animal biosafety level 2 facilities.

Analyses of morbidity and mortality - R-W1 mice (5-10, depending on availability for each treatment) were inoculated by dorsal injections of 3,000-5,000 p.f.u./0.1 mL with the SLEV strains mentioned above, in three independent experiments. Animals inoculated with $100 \mu \mathrm{L}$ phosphate buffer saline were used as control. All mice were observed during three weeks for (i) mortality percentage $(\% \mathrm{M})$, (ii) morbidity percentage (\%Mo), taking different signs of illness (excitability, tremor, laterality on gait and convulsion) as indicators, (iii) infection percentage (\%I), calculated based on brain titration of dead mice and neutralising antibodies from sera of surviving animals, (iv) time range of death, i.e., period between first and last recorded deaths, (v) mean time to death (MTD) measured in days, (vi) time range from the onset of symptoms (TROS), period of symptom manifestation in inoculated animals, and (vii) mean time from the onset of symptoms (MTOS), expressed in days. Infection of dead animals was confirmed by the detection of viral particles via standard Vero cell plaque assays (Contigiani \& Sabattini 1977). The blood of all surviving mice was collected and sera (1:5 in MEM) and were tested for neutralising antibodies using the plaquereduction neutralisation test (Early et al. 1967).

Lethal infection indices (LI) - Groups of five R-W1 mice (10 and 21-day old) were inoculated by dorsal subcu- taneous injections with serial dilutions of SLEV in $0.1 \mathrm{~mL}$ of MEM (from pure stock to $1 \times 10^{-6}$ ). Animal mortality was recorded during 19 consecutive days to calculate the LI [expressed as $\log _{10} \mathrm{PFU} / 50 \%$ lethal dose $\left.\left(\mathrm{LD}_{50}\right)\right]$, which expresses the logarithm of p.f.u. required to cause $50 \%$ death in the animals inoculated (Bradish et al. 1971).

Statistical analyses - Differences between proportions (normal approximation) were tested to compare $\% \mathrm{M}, \% \mathrm{Mo}$ and $\% \mathrm{I}$ among age groups; the tests were made for each individual viral strain and among strains. The $t$ test for differences between means was performed to detect significant differences $(p<0.05)$ in the MTD and in the MTOS among age groups (within each strain) and among strains (within each age group). All the analyses were performed using the Stata 12.0 software package (StataCorp 2009).

The association between the variables "strain" and "age" was evaluated using the Pearson's chi-square test. LI were estimated for the three SLEV strains using the Reed and Muench (1938) mathematical method.

Ethics - All procedures were carried out following the guidelines of the Institutional Committee for the Care and Use of Laboratory Animals from the School of Medicine of the National University of Córdoba, in accordance with the National Institutes of Health (USA) Guidelines for the Care and Use of Laboratory Animals and the European Economic Community Directive 86/609. The Institutional Committee approved the experimental procedures involving animals described in our study (identification 02/13-674/09).

\section{RESULTS AND DISCUSSION}

Positive infection, signs of morbidity and animal mortality were assayed after the inoculation of seven, 10 and 21-day-old mice with the SLEV strains CbaAr4005, 78V-6507 and CorAn-9275. The uninfected control animals showed no abnormal signs throughout the entire observation period. In general, animals inoculated with the different SLEV strains showed a differential response to infection. Infection was confirmed in all the animals inoculated with strain CbaAr-4005 (A in Figure). Strain 78V-6507 reached higher \%I in seven-10-day-old animals than in 21-day-old mice $(p=0.00701)$, whereas strain CorAn-9275 showed differences between seven-10-dayold $(\mathrm{p}<0.00001)$ individuals and between seven and 21day-old ones $(p=0.0095)$. Although genetic differences (Rodrigues et al. 2010) were reported for the studied strains, we cannot strongly associate this polymorphism with variations in their biological behaviour. However, differences could also be explained in terms of the genetically determined host vulnerability (Mashimo et al. 2002, Kimura et al. 2010). In addition, it is known that virus survival and ability to cause disease in mammalian hosts depend on the virus ability to avoid recognition and escape from the host immune response (Diamond 2009, Ye et al. 2012). Future studies are needed to identify the viral and host factors that determine the differential virulence among SLEV strains. Strain CbaAr-4005 showed the narrowest ranges from the TROS of the three strains and all the animals inoculated with strain CbaAr-4005 
exhibited signs of illness, with no significant differences among age groups in MTOS (Table I). By contrast, 21day-old animals inoculated with strain CorAn-9275 did not exhibit symptoms at all and a remarkably low percentage was observed for strain 78V-6507. For strain CorAn9275 , the onset of symptoms in seven-day-old mice was recorded later than in 10 -day-old animals $(\mathrm{p}<0.00001)$. The $t$ test means comparison of the three strains within each age group showed that seven-day-old $(p=0.0473)$ and 10-day-old $(\mathrm{p}<0.00001)$ individuals had earlier manifestation of symptom with strains CbaAr-4005 and 78V-6507 than with CorAn-9275.

In addition, strain CbaAr-4005 produced the highest $\%$ Mo of the three strains $(p<0.00001)$ and no significant differences among age groups were observed (B in Figure). For strain 78V-6507, a higher percentage of morbid animals was detected within seven and 10day-old mouse groups than in 21-day-old individuals ( $p$ $<0.00001$ ), whereas the highest morbidity with strain

A
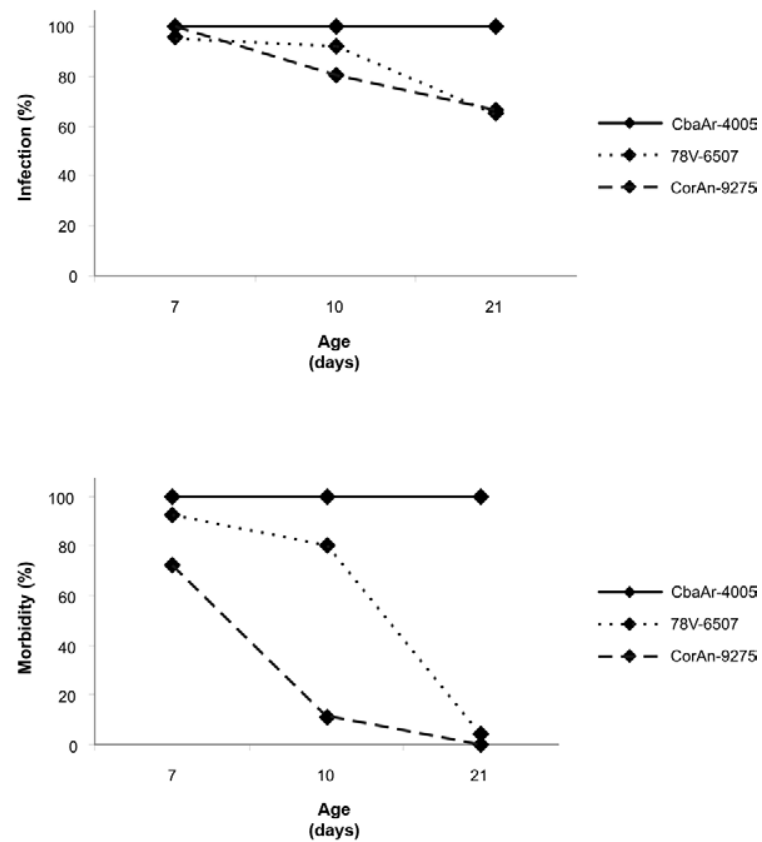

C
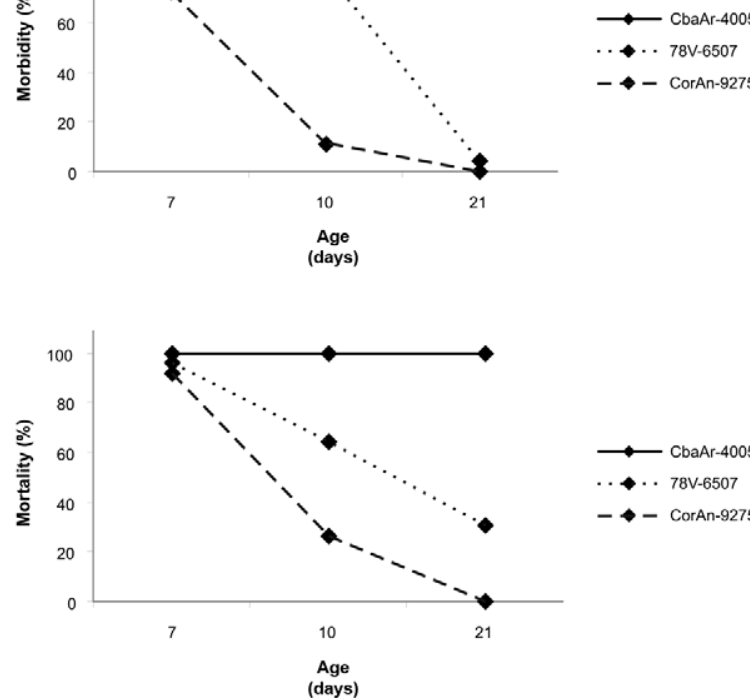

- CorAn-9275
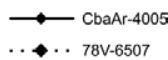

$-\bullet-$ CorAn-9275

Percentages of infection (A), morbidity (B) and mortality (C) per age group. Mice were infected with Saint Louis encephalitis virus strains. Seven, 10 and 21-day-old animals were inoculated with 3,000-5,000 plaque forming units per millilitres. Data are representative of at least three independent experiments.
CorAn-9275 occurred in seven-day-old animals $(\mathrm{p}=$ 0.0003 ). In accordance with the \%Mo, strain CbaAr4005 caused $100 \%$ of mortality in the three age groups, whereas this $\% \mathrm{M}$ tended to decrease with increasing age of animals infected with the other two strains (C in Figure). The fact that the $\% \mathrm{Mo}$ was lower than the $\% \mathrm{M}$ in 10-day-old animals inoculated with CbaAn-9275 may be due to the occurrence of undetectable symptoms. The influence of age on the development of arboviral infections has been documented in previous studies using different models. Overall, it was observed that older animals generated lower viraemias than younger animals (Andersen \& Hanson 1974, Trent et al. 1980, Reisen 2003). Bowen et al. (1980) observed that sparrows six-10 days of age were generally more susceptible to viraemic infection with SLEV than adult birds. In addition, Mahmood et al. (2004) demonstrated that nestling mourning doves inoculated with SLEV produced higher viraemia titres than adult doves that showed minimal viraemia responses after inoculation. In our study, for strains 78V-6507 and CorAn-9275, significant differences were detected between seven-10-day-old mice ( $p=0.0032$ and $p=0.0435$, respectively) and between seven-21-day-old animals ( $\mathrm{p}$ $<0.00001$ for both strains), with CorAn-9275 being the least lethal one. Our results are in agreement with those obtained by Monath et al. (1980), who conducted biological characterisation assays of SLEV in rodents. The authors observed that strain 78V-6507 caused much higher virulence than strain CorAn-9275. It is remarkable that in our assays, strain CbaAr- 4005 caused the highest mortality in 10 and 21-day-old individuals of the three strains ( $p$ $<0.00001$ ). This is in agreement with the results obtained by Díaz et al. (2011), who demonstrated that the epidemic strain $\mathrm{CbaAr}-4005$ was more viraemogenic in an avian model than other non-epidemic SLEV strains.

No differences were observed in the MTD of animals inoculated with CbaAr-4005 and 78V-6507, in any of the three studied ages. By contrast, for strain CorAn-

TABLE I

Analyses of morbidity and mortality

\begin{tabular}{lccccc}
\hline Strain & $\begin{array}{c}\text { Mice age } \\
\text { (days) }\end{array}$ & $\begin{array}{c}\text { TROS } \\
\text { (DPI) }\end{array}$ & $\begin{array}{c}\text { MTOS } \\
\text { (DPI) }\end{array}$ & $\begin{array}{c}\text { RTD } \\
\text { (DPI) }\end{array}$ & $\begin{array}{c}\text { MTD } \\
\text { (DPI) }\end{array}$ \\
\hline 78V-6507 & 7 & $5-10$ & 6 & $6-11$ & 8.04 \\
& 10 & $5-10$ & 5.9 & $6-12$ & 9.12 \\
CorAn-9275 & 21 & 12 & 12 & $7-13$ & 9 \\
& 7 & $6-13$ & 7.9 & $6-20$ & 12.4 \\
& 10 & $10-15$ & 12.5 & $13-19$ & 15 \\
CbaAr-4005 & 7 & - & - & - & - \\
& 10 & $6-7$ & 6.5 & $8-9$ & 8.05 \\
& 21 & $5-6$ & 5.7 & $6-9$ & 7.6 \\
& & $5-9$ & 6.4 & $7-11$ & 7.8
\end{tabular}

data are representative of at least three independent experiments. DPI: day post-inoculation; MTD: mean time to death; MTOS: mean time from the onset of symptoms; TRD: time range of death; TROS: time range from the onset of symptoms. 
TABLE II

Lethal infection indices (LI) in 10 and 21-day-old mice inoculated with Saint Louis encephalitis virus (SLEV) strains

\begin{tabular}{|c|c|c|c|c|c|c|}
\hline & \multicolumn{2}{|c|}{ CbaAr-4005 } & \multicolumn{2}{|c|}{$78 \mathrm{~V}-6507$} & \multicolumn{2}{|c|}{ CorAn-9275 } \\
\hline & 10 days & 21 days & 10 days & 21 days & 10 days & 21 days \\
\hline LI & 0.02 & 0.30 & 1.75 & 3.99 & 3.90 & 5.08 \\
\hline $\log _{10}$ p.f.u. $/ \mathrm{LD}_{50}$ & $5.30 / 5.28$ & $5.30 / 5.00$ & $6.07 / 4.32$ & $6.07 / 2.08$ & $6.30 / 2.40$ & $6.30 / 1.22$ \\
\hline Antilog LI (p.f.u.) & 1.0471 & 1.9998 & 56.23 & 9772.3 & 7943.28 & 120226.4 \\
\hline
\end{tabular}

the LI were obtained by Reed and Muench (1938) analysis and is expressed as the titre of the stock divided by the dilution needed to reach a $50 \%$ lethal dose $\left(\mathrm{LD}_{50}\right)$. p.f.u.: plaque forming units.

9275, seven-day-old animals showed a lower MTD than 10 -day-old animals $(p=0.0007)$. The comparison of the three strains together showed that the highest MTD was observed in seven and 10-day-old mice inoculated with strain CorAn-9275 ( $\mathrm{p}<0.00001)$. Of the two strains that caused mortality in 21-day-old animals, 78V-6507 showed the highest MTD $(p=0.0102)$ (Table I).

Table II shows the calculated values of LI $\left(\mathrm{LD}_{50}\right)$ of 10 and 21-day-old animals inoculated with the SLEV three strains (7-day-old mice were not included because no mortality/morbidity differences were observed within this group). It was necessary to inoculate only 1 p.f.u. and 2 p.f.u. in $0.1 \mathrm{~mL}$ (in 10 and 21-day-old mice, respectively) to cause $50 \%$ mortality with the epidemic strain CbaAr-4005, which proved to be the most lethal of the three variants. The number of p.f.u. for a $\mathrm{LD}_{50}$ increased with increasing age of the animals, when inoculated with the other two strains. These results are in agreement with those obtained in the essays of mortality and morbidity and they prove a strong association between the physical effects caused by SLEV strains and the age of inoculated animals. The number of p.f.u for $\mathrm{LD}_{50}$ was markedly higher with CorAn-9275 than with any of the other strains.

Although the silent circulation of SLEV (genotypes II, V and VII) in Córdoba city was confirmed prior the 2005 outbreak (Díaz et al. 2012), only the newly introduced strain CbaAr-4005 (genotype III) was isolated during the mentioned outbreak (Spinsanti et al. 2008). In the present study we found that SLEV CbaAr-4005 was the most virulent strain for Swiss albino mice. It is well known that the introduction and geographical spread of new strains can cause the emergence and reemergence of mosquito-borne viruses in different regions (Mackenzie et al. 2004). It is worth pointing out that this study is dependent on single isolate that may or may not be representative of all the circulating variants of each genotype. In such scenario, our results highlight the importance of performing biological characterisations of circulating arbovirus strains that could be responsible for emerging or reemerging human diseases. However, in a comprehensive epidemiological evaluation, other aspects, such as amplification capacity in avian hosts, should be considered carefully. Díaz et al. (2012) showed that SLEV CbaAr-4005 reached higher titres in 24-h-old chicken than CorAn-9275 and 78V-6507. Moreover, variations in competence among different hosts for SLEV have direct implications in the quantity of virus ingested by mosquito vectors from the viraemic avian host, determining the transmission rates by the vector. Future studies of our research group will analyse the contribution of rodents to the maintenance cycle of different SLEV strains. We will also perform detailed analyses concerning neuroinvasiveness and viraemia levels during the course of infection, using a murine model. Molecular tools, such as infectious clones, are necessary to study the possible genetic determinants involved in differential pathogenesis of the different strains.

\section{ACKNOWLEDGEMENTS}

To Martín Lépez, for technical assistance in the maintenance of laboratory animals, and to Dr Pilar Díaz and Sonia Pou, FCM-UNC, for their support with the statistical analyses.

\section{REFERENCES}

Andersen AA, Hanson RP 1974. Influence of sex and age on natural resistance to St. Louis encephalitis virus infection in mice. Infect Immunity 9: 1123-1125.

Bowen GS, Monath TP, Kemp GE, Kershner JH, Kirk LJ 1980. Geographic variation among St. Louis encephalitis virus strains in the viremic responses of avian hosts. Am J Trop Med Hyg 29: 1411-1419.

Bradish CJ, Allner K, Maber HB 1971. The virulence of original and rived strains of Smeliki Forest virus for mice, guinea-pigs and rabbits. J Gen Virol 12: 141-160.

Contigiani MS, Sabattini MS 1977. Virulencia diferencial de cepas de virus Junín por marcadores biológicos en ratones y cobayos. Medicina (B Aires) 37: 244-251.

Diamond MS 2009. Mechanisms of evasion of the type I interferon antiviral response by flaviviruses. J Interferon Cytokine Res 29: 521-530.

Díaz LA 2009. Patrones de actividad y estacionalidad del virus St. Louis encephalitis en Córdoba, Argentina, $\mathrm{PhD}$ Thesis, Nacional University of Córdoba, Córdoba, 193 pp.

Díaz LA, Albrieu-Llinás G, Vázquez A, Tenorio A, Contigiani MS 2012. Silent circulation of St. Louis Encephalitis virus prior to an encephalitis outbreak in Córdoba, Argentina (2005). PLoS Negl Trop Dis 6: 1489.

Díaz LA, Nemeth NM, Bowen RA, Almirón WR, Contigian MS 2011. Comparison of Argentinean Saint Louis encephalitis virus non- 
epidemic and epidemic strain infections in an avian model. PLoS Negl Trop Dis 5: 1177.

Díaz LA, Occelli M, Almeida FL, Almirón WR, Contigiani MS 2008. Eared dove (Zenaida auriculata, Columbidae) as host for St. Louis encephalitis virus (Flaviviridae, Flavivirus). Vector Borne Zoonotic Dis 8: 277-282.

Díaz LA, Ré V, Almirón WR, Farías A, Vázquez A, Sánchez-Seco MP, Aguilar J, Spinsanti L, Konigheim B, Visintin A, García J, Morales MA, Tenorio A, Contigiani MS 2006. Genotype III Saint Louis encephalitis virus outbreak in Argentina, 2005. Emerg Infect Dis 12: 1752-1754.

Early E, Peralta PH, Johnson KM 1967. A plaque neutralization method for arboviruses. Proc Soc Exp Biol Med 25: 741-747.

Kimura T, Sasaki M, Okumura M, Kim E, Sawa H 2010. Flavivirus encephalitis: pathological aspects of mouse and other animal models. Vet Pathol 47: 806-818.

Mackenzie JS, Gubler DJ, Petersen LR 2004. Emerging flaviviruses: the spread and resurgence of Japanese encephalitis, West Nile and dengue viruses. Nat Med 10 (Suppl. 12): S98-S109.

Mahmood F, Chiles RE, Fang Y, Barker CM, Reisen WK 2004. Role of nestling mourning doves and house finches as amplifying hosts of St. Louis encephalitis virus. J Med Entomol 41: 965-972.

Mashimo T, Lucas M, Simon-Chazottes D, Frenkiel MP, Montagutelli X, Ceccaldi P, Deubels V, Guénet JL, Després P 2002. A nonsense mutation in the gene encoding 2'-5'-oligoadenylate synthetase/L1 isoform is associated with West Nile virus susceptibility in laboratory mice. PNAS 99: 11311-11316.

Mitchell CJ, Gubler DJ, Monath TP 1983. Variation in infectivity of Saint Louis encephalitis viral strains for Culex pipiens quinquefasciatus (Diptera: Culicidae). J Med Entomol 20: 526-533.

Monath TP 1980. Epidemiology. In TP Monath, St. Louis encephalitis, American Public Health Association, Washington DC, p. 239.

Monath TP, Cropp CB, Bowen GS, Kemp GE, Mitchell CJ, Gardner JJ 1980. Variation in virulence for mice and rhesus monkeys among St. Louis encephalitis virus strains of different origin. Am J Trop Med Hyg 29: 948-962.

Reed LJ, Muench H 1938. A simple method of estimating fifty per cent endpoints. Am J Hyg 27: 493-497.
Reisen WK 2003. Epidemiology of St. Louis encephalitis virus. $A d v$ Virus Res 61: 139-183.

Rocco IM, Santos CL, Bisordi I, Petrella SM, Pereira LE, Souza RP, Coimbra TL, Bessa TAF, Oshiro FM, Lima LBQ, Cerroni MP, Marti AT, Barbosa VM, Katz G, Suzuki A 2005. St. Louis encephalitis virus: first isolation from a human in Sao Paulo state, Brazil. Rev Inst Med Trop Sao Paulo 47: 281-285.

Rodrigues SG, Nunes MR, Casseb SM, Prazeres AS, Rodrigues DS, Silva MO, Cruz AC, Tavarez-Neto JC, Vasconcelos PF 2010. Molecular epidemiology of Saint Louis encephalitis virus in the Brazilian Amazon: genetic divergence and dispersal. J Gen Virol 91: 2420-2427.

Sabattini MS, Avilés G, Monath TP 1998. Historical, epidemiological and ecological aspects of arbovirus in Argentina: Flaviviridae, Bunyaviridae and Rhabdoviridae. In APAT da Rosa, PFC Vasconcelos, JFST da Rosa (eds.), An overview of arbovirology in Brazil and neighboring countries, Instituto Evandro Chagas, Belém, p. 113-134.

Seijo A, Morales A, Poustis G, Romero Y, Efron E, Vilora G, Lloveras S, Giamperetti S, Puente T, Monroiq J, Luppo V, Enría D 2011. Outbreak of St. Louis encephalitis in the metropolitan Buenos Aires Area. Medicina (B Aires) 71: 211-217.

Spinsanti L, Basquiera A, Bulacio S, Somale V, Kim SCH, Ré V, Rabbat D, Zárate A, Zlocowski JC, Mayor CQ, Contigiani M, Palacio S 2003. St. Louis encephalitis in Argentina: the first case reported in the last seventeen years. Emerg Infect Dis 9: 271-273.

Spinsanti LI, Díaz LA, Glatstein N, Arzelán S, Morales MA, Farías AA, Fabbri C, Aguilar JJ, Ré V, Farías M, Almirón WR, Hunsperger E, Siirin M, da Rosa AT, Tesh RB, Enría D, Contigiani M 2008. Human outbreak of St. Louis encephalitis detected in Argentina, 2005. J Clin Virol 42: 27-33.

StataCorp 2009. Stata Statistical Software: release 11, College Station, TX: StataCorp LP.

Trent DW, Monath TP, Bowe GS, Vordan AV 1980. Variation among strains of St. Louis encephalitis virus: basis for genetic, pathogenetic and epidemiologic classification. Ann NY Acad Sci 354: 219-237.

Ye J, Zhu B, Fu ZF, Chen H, Cao S 2012. Immune evasion strategies of flaviviruses. Vaccine 31: 461-471. 